\title{
BIHOLOMORPHIC INVARIANTS OF A HYPERBOLIC MANIFOLD AND SOME APPLICATIONS
}

\author{
BY
}

\author{
B. L. FRIDMAN
}

\begin{abstract}
A biholomorphically invariant real function $h_{x}$ is defined for a hyperbolic manifold $X$. Properties of such functions are studied. These properties are applied to prove the following theorem. If a hyperbolic manifold $X$ can be exhausted by biholomorphic images of a strictly pseudoconvex domain $D \subset \mathbf{C}^{n}$ with $\partial D \in C^{3}$, then $X$ is biholomorphically equivalent either to $D$ or to the unit ball in $\mathbf{C}^{n}$. The properties of $h_{D}$ are also applied to some questions concerning the group of analytical automorphisms of a strictly pseudoconvex domain and to similar questions concerning polyhedra.
\end{abstract}

Introduction. Let $X$ be a hyperbolic manifold of complex dimension $n$. Let $\mathcal{H} \subset \mathbf{C}^{n}$ be a bounded homogeneous domain. We denote by $B\left(x_{0}, r\right)$ the ball in Kobayashi metric (see [9]) of radius $r>0$ with center at $x_{0} \in X$. By $\Re$ we denote the set of all $r$ such that there exists a biholomorphic imbedding $F: \mathcal{H} \rightarrow X, F(\mathcal{H}) \supset$ $B\left(x_{0}, r\right)$. We set

DEFINITION 0.1.

$$
h_{X}\left(x_{0}, \mathcal{H}\right)=\inf _{r \in \Re} 1 / r .
$$

Clearly this function is biholomorphically invariant: namely, if $\Phi: X \rightarrow Y$ is biholomorphic, then $h_{X}=h_{Y} \circ \Phi$.

Such functions with the property of being invariants could be defined for any holomorphically invariant metric and any homogeneous domain $\mathcal{H}$. For the Carathéodory metric and $\mathcal{K}=U^{n}$ the unit polydisk, these functions were defined and studied in [4]. In this article we will consider these functions on strictly pseudoconvex domains. The metric we will consider here is the Kobayashi metric, $\mathcal{H}$ will generally be $B^{n}$, the unit ball. We will prove that $h_{X}$ is nonnegative and continuous, and if $h_{X}\left(x_{0}, \mathcal{K}\right)=0$ at a point $x_{0} \in X$ where $X$ is a hyperbolic manifold, then $h_{X} \equiv 0$ and $X$ is biholomorphically equivalent to $\mathcal{H}$.

Let $\left\{X_{k}\right\}, 1 \leqslant k<\infty$, be a sequence of subdomains of $X$.

Definition 0.2 . We will say that $\left\{X_{k}\right\}$ exhausts $X$ if for any compact $K \subset X$ there exists a number $N$ such that $X_{n} \supset K$ for any $n>N$.

One of the properties of $h_{X}$ is that $h_{X_{n}} \rightarrow h_{X}$ uniformly on compacta if $X$ is completely hyperbolic. (A hyperbolic manifold is said to be complete if all subsets bounded in the Kobayashi metric are relatively compact in $X$.)

Received by the editors February 24, 1982. Portions of this paper were presented at 85 th Summer Meeting of the AMS, Pittsburgh, Pennsylvania, August 1981.

1980 Mathematics Subject Classification. Primary 32H20, 32F 15. 
Let $h_{D}(z)=h_{D}\left(z, B^{n}\right)$ where $D$ is a bounded strictly pseudoconvex domain with $\partial D \in C^{3}$. The main property of this function is

$$
h_{D}(z) \underset{z \rightarrow \partial D}{\rightarrow} 0 .
$$

This property shows for instance that the only bounded strictly pseudoconvex domain where $h_{D}$ is a constant is the unit ball.

We will apply the properties of these functions to several questions. The first one is the following.

Let $G$ be a bounded domain in $\mathbf{C}^{n}, X$ a manifold. We will say that $X$ can be exhausted by biholomorphic images of $G$ (or shorter "by $G$ ") if for any compact $K \subset X$ there is a biholomorphic imbedding $F_{K}: G \rightarrow X$ such that $F_{K}(G) \supset K$.

Suppose that $X$ is a hyperbolic manifold and $X$ can be exhausted by $G$. The question is to describe $X$.

Questions similar to this one were studied in [1-4]. In [2] it was shown, in particular, that if a hyperbolic manifold $X$ admits an exhaustion by an increasing sequence of biholomorphic images of the ball $B^{n}$ (or $U^{n}$ ), then $X$ is biholomorphically equivalent to $B^{n}$ (or $U^{n}$ ). In this theorem $B^{n}$ may be replaced by any bounded homogeneous domain (see Theorem 1.2 below). In [1] sharp estimates were obtained for the best imbedding of a polydisc in a ball and a ball in a polydisc. In terms of our functions these results are

$$
h_{B^{n}}\left(x, U^{n}\right)=h_{U^{n}}\left(x, B^{n}\right) \equiv(\operatorname{In}[(\sqrt{n+1}) /(\sqrt{n-1})])^{-1} .
$$

In the direction of this question we obtain the following theorem.

THEOREM I. Let $D \subset \mathbf{C}^{n}$ be a bounded strictly pseudoconvex domain with boundary of class $C^{3}$, and $X$ is a completely hyperbolic manifold of complex dimension $n$. Suppose it is possible to exhaust $X$ by biholomorphic images of $D$. Then either $X$ is biholomorphically equivalent to $D$ or $X$ is biholomorphically equivalent to $B^{n}$.

As a corollary we obtain

THEOREM II. Two bounded strictly pseudoconvex domains with boundaries of class $C^{3}$ are biholomorphically equivalent if and only if each of them can be exhausted by the other.

To put it another way, if two such domains are "approximately" equivalent they are biholomorphically equivalent.

Theorems I and II were announced in [4].

Evidently any orbit of the group $\operatorname{Aut}(D)$ of analytical automorphisms of a domain $D$ lies on a level line of $h_{D}$. So $h_{D}$ may give us some information about $\operatorname{Aut}(D)$. We obtain a simple proof of the following theorem: if $D$ is a bounded strictly pseudoconvex domain such that $\operatorname{Aut}(D)$ is noncompact, then $D$ is biholomorphically equivalent to a ball. This theorem is very well known for $\partial D \in C^{\infty}$ (see [11]). We prove it for $\partial D \in C^{3}$. One more generalization of this theorem can be found in $\S 4$. We prove there one theorem concerning $\operatorname{Aut}(G)$ where $G$ is a polyhedron.

In $\$ 1$ we prove Theorem 1.2 about exhausting a hyperbolic manifold by a homogeneous domain. $\$ 2$ is devoted to showing the relation between functions $h_{X_{n}}$ 
and $h_{X}$ when $X$ is exhausted by $\left\{X_{n}\right\}$. In $\S 3$ we prove property $(0.2)$. In $\$ 4$ we present the proofs of Theorems I, II, theorems concerning $\operatorname{Aut}(D)$ and some remarks.

I wish to express my deep gratitude to Robert Bix who gave me much help in preparing this paper for publication.

1. Exhausting by biholomorphic images of a homogeneous domain. We begin with a general lemma.

LEMMA 1.1. Let $D \subset \mathbf{C}^{n}$ be a bounded domain, $X$ a hyperbolic manifold of complex dimension $n$. Suppose that there exist two relatively compact sets $K_{1} \subset D$ and $K_{2} \subset X$ and a sequence $\left\{F_{k}\right\}$ of mappings $F_{k}: D \rightarrow X$ that satisfy the following conditions:

(1) $F_{k}: D \rightarrow F_{k}(D)$ is biholomorphic for any $k<\infty$.

(2) For any $1 \leqslant k<\infty$ there exists a point $z_{k} \in K_{1}$ such that $F_{k}\left(z_{k}\right) \in K_{2}$.

(3) For any compact $K \subset X$ there exists a number $s=s(K)$ such that $F_{s}(D) \supset K$.

Then $X$ is biholomorphically equivalent to $D$.

Proof. (1) We denote $\Phi_{k}=F_{k}^{-1}: F_{k}(D) \rightarrow D, x_{k}=F_{k}\left(z_{k}\right)$. Let $U_{1} \Subset U_{2} \Subset$ $\cdots \Subset U_{n} \Subset \cdots$ be a sequence of open sets such that $\cup U_{n}=X$. One can see that by passing to a subsequence if necessary we may assume that $\left\{\Phi_{k}\right\}$ satisfies the following condition: for each $n \geqslant 1$, there exists an $N$ such that $\left\{\Phi_{k}\right\}_{k \geqslant N}$ is defined on $U_{n}$ and converges uniformly on compacta in $U_{n}$ to $\Phi: U_{n} \rightarrow \bar{D}$. Evidently, $\Phi$ is defined on $X$.

(2) Evidently $\Phi(X) \subset \bar{D}$. We want to show that $\Phi(X) \subset D$. Since $\left\{x_{k}\right\} \subset K_{2}$ and $\left\{z_{k}\right\} \subset K_{1}$, we may assume (by passing to a subsequence if necessary) that $\left\{x_{k}\right\}$ converges to $x_{0} \in \bar{K}_{2}$ and $\left\{z_{k}\right\}$ to $z_{0} \in \bar{K}_{1}$. One can easily see that $\Phi\left(x_{0}\right)=z_{0}$. Let $\varepsilon>0$ be so small that the ball (in Kobayashi metric) $B_{X}\left(x_{0}, \varepsilon\right) \Subset X$, and $B_{D}\left(z_{0}, \varepsilon\right)$ $\Subset D$. Let $z \in B_{D}\left(z_{0}, \varepsilon / 2\right)$. For a large number $k, F_{k}\left(B_{D}\left(z_{0}, \varepsilon / 2\right)\right) \subset B_{X}\left(x_{0}, \varepsilon\right)$ (we use here the important property of Kobayashi's metric: $\rho\left(F_{k}\left(x^{\prime}\right), F_{k}\left(x^{\prime \prime}\right)\right) \leqslant$ $\left.\rho\left(x^{\prime}, x^{\prime \prime}\right)\right)$. So, for this number $k, F_{k}(z)=x_{k}^{\prime} \in B_{X}\left(x_{0}, \varepsilon\right)$. If $x^{\prime}$ is the limit point of $\left\{x_{k}^{\prime}\right\}$ one can see that $\Phi\left(x^{\prime}\right)=z$. This means that $\Phi(X) \supset B_{D}\left(z_{0}, \varepsilon / 2\right) . \Phi$ is a limit of regular holomorphic mappings. Since $X$ is connected $\Phi$ may be either regular at any point at $X$ or the Jacobian of $\Phi$ (in local coordinates) vanishes on $X$.

In the latter case $\Phi(X)$ could not contain any open set (by Sard's theorem). Since $\Phi(X) \supset B_{D}\left(z_{0}, \varepsilon / 2\right), \Phi$ is regular on $X$. This implies that $\Phi(X)$ is open, so $\Phi(X) \subset D$.

(3) We will show now that $\Phi$ is one-to-one. Let $x^{\prime}, x^{\prime \prime} \in X$. For a large number $k$ and the Kobayashi metric $\rho$ we have

$$
\begin{aligned}
\rho\left(x^{\prime}, x^{\prime \prime}\right) & =\rho\left(F_{k} \circ \Phi_{k}\left(x^{\prime}\right), F_{k} \circ \Phi_{k}\left(x^{\prime \prime}\right)\right) \leqslant \rho\left(\Phi_{k}\left(x^{\prime}\right), \Phi_{k}\left(x^{\prime \prime}\right)\right) \\
& \leqslant \rho\left(\Phi_{k}\left(x^{\prime}\right), \Phi\left(x^{\prime}\right)\right)+\rho\left(\Phi\left(x^{\prime}\right), \Phi\left(x^{\prime \prime}\right)\right)+\rho\left(\Phi\left(x^{\prime \prime}\right), \Phi_{k}\left(x^{\prime \prime}\right)\right) .
\end{aligned}
$$

When $k \rightarrow \infty$ we obtain

$$
\rho\left(x^{\prime}, x^{\prime \prime}\right) \leqslant \rho\left(\Phi\left(x^{\prime}\right), \Phi\left(x^{\prime \prime}\right)\right) .
$$

Hence, if $\Phi\left(x^{\prime}\right)=\Phi\left(x^{\prime \prime}\right)$, then $x^{\prime}=x^{\prime \prime}$.

(4) We will show that $\Phi(X) \supset D$. From all we have already proved, $X$ is biholomorphically equivalent to a domain $\Phi(X) \subset D$. From this point on we can 
consider $X$ as a bounded domain in $\mathbf{C}^{n}$. We may now assume that $\left\{F_{k}\right\}$ converges uniformly on compacta to $F: D \rightarrow \bar{X}$. It follows as in steps (1)-(3) that $F\left(z_{0}\right)=x_{0}$ and $F(D) \subset X$. For the mapping $\Phi \circ F: D \rightarrow D$ and any $z \in D$ we have

$$
\Phi \circ F(z)=\lim _{k \rightarrow \infty} \Phi_{k} \circ F_{k}(z)=z .
$$

Hence $\Phi(X) \supset D$.

It follows from (1)-(4) that $\Phi: X \rightarrow D$ is a biholomorphism, completing the proof of the lemma.

TheOREM 1.2. Let $\mathcal{H} \subset \mathbf{C}^{n}$ be a bounded homogeneous domain, and let $X$ be a hyperbolic manifold of complex dimension $n$. If $X$ can be exhausted by biholomorphic images of $\mathcal{H}$, then $X$ is biholomorphically equivalent to $\mathcal{H}$.

Proof. Let $\Phi_{k}: \mathcal{H} \rightarrow X$ be a sequence of biholomorphic mappings such that for any compact $K \subset X$ there exists a number $s$ such that $\Phi_{s}(\mathcal{H}) \supset K$. Let $x_{0} \in X$ and $z_{0} \in \mathcal{H}$. Without loss of generality, we may suppose that $x_{0} \in \Phi_{k}(\mathcal{H})$ for any $k$, $1 \leqslant k<\infty$. Since our domain is homogeneous, we can find $\psi_{k} \in \operatorname{Aut}(\mathcal{H})$ such that $\psi_{k}\left(\Phi_{k}^{-1}\left(x_{0}\right)\right)=z_{0}$. Now the sequence $F_{k}=\Phi_{k} \circ \psi_{k}^{-1}$ satisfies all the conditions of Lemma 1.1 for $K_{1}=\left\{z_{0}\right\}$ and $K_{2}=\left\{x_{0}\right\}$. Applying this lemma we find that $X$ is biholomorphically equivalent to $\mathcal{H}$.

THEOREM 1.3. Let $X$ be a connected hyperbolic manifold, $\operatorname{dim}_{\mathrm{C}} X=n$, and let $\mathcal{H C} \subset \mathbf{C}^{n}$ be a bounded homogeneous domain. Then

(1) $h_{X}(x)=h_{X}(x, \mathcal{H})$ is continuous on $X$ and $h_{X} \geqslant 0$.

(2) If there is an $x_{0} \in X$ such that $h_{X}\left(x_{0}\right)=0$, then $h_{X}(x) \equiv 0$ and $X$ is biholomorphically equivalent to $\mathcal{H}$.

Proof. (1) If there exists an $x_{0} \in X$ such that $h_{X}\left(x_{0}\right)=0$ by Definition 0.1 , there exists a sequence $\left\{\Phi_{k}\right\}, \Phi_{k}: \mathcal{H} \rightarrow X$, such that $\Phi_{k}(\mathcal{H}) \supset B_{X}\left(x_{0}, k\right)$. Now applying Theorem 1.2 we obtain that $X$ is biholomorphically equivalent to $\mathcal{H}$ and, therefore, $h_{X} \equiv 0$.

(2) Let $h_{X}\left(x_{0}\right)>0$ at any $x_{0} \in X$. Let us consider $H_{X}\left(x_{0}\right)=1 / h_{X}(x)=\sup _{r \in \mathscr{R}} r$ (see Definition 0.1). It is enough to prove that $H_{X}$ is continuous. Let $x_{1}, x_{2} \in X$ be so close that

$$
2 \rho\left(x_{1}, x_{2}\right)<H_{X}\left(x_{1}\right) .
$$

Let $\varepsilon>0$ be any number such that

$$
H_{X}\left(x_{1}\right)-2 \rho\left(x_{1}, x_{2}\right)-2 \varepsilon>0 .
$$

Since $\rho$ is a metric, one can obtain

$$
B_{X}\left(x_{1}, H_{X}\left(x_{1}\right)-\varepsilon\right) \supset B_{X}\left(x_{2}, H_{X}\left(x_{1}\right)-\varepsilon-\rho\left(x_{1}, x_{2}\right)\right) .
$$

This means that

$$
H_{X}\left(x_{2}\right) \geqslant H_{X}\left(x_{1}\right)-\varepsilon-\rho\left(x_{1}, x_{2}\right)
$$

So

$$
H_{X}\left(x_{1}\right)-H_{X}\left(x_{2}\right) \leqslant \rho\left(x_{1}, x_{2}\right)+\varepsilon
$$


and

$$
H_{X}\left(x_{2}\right)-\varepsilon-\rho\left(x_{1}, x_{2}\right)>0 .
$$

Similarly

$$
B_{X}\left(x_{2}, H_{X}\left(x_{2}\right)-\varepsilon\right) \supset B_{X}\left(x_{1}, H_{X}\left(x_{2}\right)-\varepsilon-\rho\left(x_{1}, x_{2}\right)\right)
$$

and

$$
H_{X}\left(x_{2}\right)-H_{X}\left(x_{1}\right) \leqslant \rho\left(x_{1}, x_{2}\right)+\varepsilon .
$$

From (1.1) and (1.2), letting $\varepsilon \rightarrow 0$ yields

$$
\left|H_{X}\left(x_{2}\right)-H_{X}\left(x_{1}\right)\right| \leqslant \rho\left(x_{1}, x_{2}\right) \text {. }
$$

This inequality shows that $H_{X}$ is continuous in the topology induced by the Kobayashi metric. Since this topology is the usual one, we have finished the proof of Theorem 1.3.

2. Exhaustion and the behaviour of $h_{X}$. The main theorem we will prove in this section is the following one. $\left(h_{X}\right.$ below means $h_{X}\left(x, B^{n}\right)$.)

THEOREM 2.1. Let $X$ be a completely hyperbolic manifold and let $\left\{X_{n}\right\}, 1 \leqslant n<\infty$, be a sequence of subdomains that exhausts $X$. Then, for any $x_{0} \in X$,

$$
\lim _{n \rightarrow \infty} h_{X_{n}}\left(x_{0}\right)=h_{X}\left(x_{0}\right) \text {. }
$$

REMARK. By Definition 0.2 there exists an $N$ such that $x_{0} \in X_{n}$ for any $n \geqslant N$. The limit in the statement above is taken to mean $n \rightarrow \infty$ for $n \geqslant N$.

Before proving Theorem 2.1, we recall the definition of Kobayashi's metric [9].

Let $M$ be a complex manifold, $p, q \in M$. Chain $\sigma$ is a set of

(1) $m$ holomorphic mappings $f_{i}: B^{1} \rightarrow M, f_{i}\left(B^{1}\right) \Subset M, i=1, \ldots, m$, and

(2) $m$ pairs of points $z_{j}, w_{j} \in B^{1}$ such that $f_{1}\left(z_{1}\right)=p, f_{m}\left(w_{m}\right)=q$, and $f_{j}\left(w_{j}\right)=$ $f_{j+1}\left(z_{j+1}\right)$ for $j=1, \ldots, m-1$. Let $d(z, w)$ denote the usual Lobachevsky-Poincaré distance between $z$ and $w$.

DEFINITION 2.2.

$$
\rho_{M}(p, q)=\inf _{\sigma} \sum_{j=1}^{m} d\left(z_{j}, w_{j}\right)
$$

where the inf is taken for all chains ( $m$ may be any number.)

The next property of this metric is particularly important. Let $M_{1}$ and $M_{2}$ be hyperbolic manifolds. If $F: M_{1} \rightarrow M_{2}$ is holomorphic, then

$$
\rho_{M_{2}}\left(F\left(x_{1}\right), F\left(x_{2}\right)\right) \leqslant \rho_{M_{1}}\left(x_{1}, x_{2}\right) \text {. }
$$

As a corollary we obtain

(2.2) If $M_{1} \subset M_{2}$, then $\rho_{M_{2}}\left(x_{1}, x_{2}\right) \leqslant \rho_{M_{1}}\left(x_{1}, x_{2}\right)$ for $x_{1}, x_{2} \in M_{1}$.

If $B_{M}\left(x_{0}, R\right)=\left\{x \mid \rho_{M}\left(x_{0}, x\right)<R\right\}$ is a ball in the Kobayashi metric on $M$, then (2.2) is equivalent to the following statement: for any $x_{0} \in M_{1}$ and $R>0$,

$$
M_{1} \subset M_{2} \Rightarrow B_{M_{1}}\left(x_{0}, R\right) \subseteq B_{M_{2}}\left(x_{0}, R\right)
$$


LEMMA 2.3. Let $X$ be any complete hyperbolic manifold and let $\left\{X_{n}\right\}_{n=1}^{\infty}$ be a sequence of submanifolds that exhausts $X$. Let $x_{0} \in X$ and $R>0$. Then for any $\varepsilon>0$ there exists an $N$ such that $B_{X_{n}}\left(x_{0}, R\right) \supset B_{X}\left(x_{0}, R-\varepsilon\right)$ for all $n>N$.

Proof. Let $\left\{U_{n}\right\}_{n=1}^{\infty}$ be a sequence of open submanifolds in $X$ such that

(i) $U_{n+1} \supseteq U_{n}$ for all $n$,

(ii) $\cup_{n=1}^{\infty} U_{n}=X$.

(1) Let $x$ be a fixed element of $X$ such that $\rho_{X}\left(x_{0}, x\right) \leqslant R-\varepsilon$. By Definition 2.2 there exists a chain $\sigma_{m}=\left\{f_{i}, z_{i}, w_{i} \mid i=1, \ldots, m\right\}$ such that

$$
\sum_{i=1}^{m} d\left(z_{i}, w_{i}\right) \leqslant R-\varepsilon / 2 .
$$

Since $\cup_{i=1}^{m} f_{i}\left(B^{1}\right) \Subset X$, we can find a number $l$ such that

$$
U_{l} \supset \bigcup_{k=1}^{m} f_{i}\left(B^{1}\right) \text {. }
$$

By Definition 2.2,

$$
\rho_{U_{l}}\left(x_{0}, x\right) \leqslant R-\varepsilon / 2<R .
$$

Thus for any point $x \in X$ satisfying $\rho_{X}\left(x_{0}, x\right) \leqslant R-\varepsilon$, we have found an $l=l(x)$ such that $x \in B_{U_{l}}\left(x_{0}, R\right)$. Since $\overline{B_{X}\left(x_{0}, R-\varepsilon\right)}$ is compact (because $X$ is complete), we can find a finite number of points $\left\{x_{i}\right\}_{i=1}^{s}$ such that

$$
\bigcup_{i=1}^{n} B_{U_{l(i)}}\left(x_{0}, R\right) \supset \overline{B_{X}\left(x_{0}, R-\varepsilon\right)},
$$

where $l(i)=l\left(x_{i}\right)$. Since the sequence $\left\{U_{n}\right\}_{n=1}^{\infty}$ is increasing (2.3) implies that

$$
B_{U_{L}}\left(x_{0}, R\right) \supset \overline{B_{X}\left(x_{0}, R-\varepsilon\right)},
$$

where $L=\max _{1 \leqslant i<s} l\left(x_{i}\right)$. There exists a number $N$ such that $X_{n} \supset U_{L}$ for $n>N$. It follows from (2.3) and (2.4) that

$$
B_{X_{n}}\left(x_{0}, R\right) \supseteq B_{U_{L}}\left(x_{0}, R\right) \supset \overline{B\left(x_{0}, R-\varepsilon\right)} .
$$

The lemma is proved.

Proof of Theorem 2.1. (1) Let $h_{X}\left(x_{0}\right)<1 / r$. There exists a biholomorphic mapping $F: B^{n} \rightarrow X$ such that $F\left(B^{n}\right) \supset B_{X}\left(x_{0}, r\right)$.

For any $\varepsilon>0$, there exists a $\delta>0$ such that $F\left((1-\delta) B^{n}\right) \supset B_{X}\left(x_{0}, r-\varepsilon\right)$. Since $F\left((1-\delta) B^{n}\right) \Subset X$, there exists a number $N$ such that $X_{K} \supset F\left((1-\delta) B^{n}\right)$ for any $K>N$. Now

$$
X_{K} \supset F\left((1-\delta) B^{n}\right) \supset B_{X}\left(x_{0}, r-\varepsilon\right) \supset B_{X_{K}}\left(x_{0}, r-\varepsilon\right),
$$

so

$$
h_{X_{K}}\left(x_{0}\right) \leqslant 1 /(r-\varepsilon) \text {. }
$$

We have proved that

$$
\limsup h_{X_{K}}\left(x_{0}\right) \leqslant 1 / r
$$


whenever $h_{X}\left(x_{0}\right)<1 / r$. Therefore

$$
\lim \sup h_{X_{K}}\left(x_{0}\right) \leqslant h_{X}\left(x_{0}\right) \text {. }
$$

(2) Let

$$
\liminf h_{X_{K}}\left(x_{0}\right)<1 / R
$$

for some $R>0$. By Lemma 2.3, for any $\varepsilon>0$ we can find an $N$ such that

$$
B_{X_{K}}\left(x_{0}, R\right) \supset B_{X}\left(x_{0}, R-\varepsilon\right)
$$

for any $K>N$. Now using (2.6) we can find a number $l>N$ such that

$$
h_{X_{l}}\left(x_{0}\right)<1 / R \text {. }
$$

Hence there exists a biholomorphic mapping $F: B^{n} \rightarrow X_{l}$ suchthat $F\left(B^{n}\right) \supset$ $B_{X_{I}}\left(x_{0}, R\right)$. Together with (2.7), this yields

$$
F\left(B^{n}\right) \supset B_{X}\left(x_{0}, R-\varepsilon\right) \text {, so } h_{X}\left(x_{0}\right) \leqslant 1 /(R-\varepsilon) .
$$

Since $\varepsilon>0$ was arbitrary,

$$
h_{X}\left(x_{0}\right) \leqslant 1 / R \text {. }
$$

Comparing this and the choice of $R$ in (2.6), we see that

$$
h_{X}\left(x_{0}\right) \leqslant \liminf h_{X_{K}}\left(x_{0}\right) \text {. }
$$

(3) From (2.5) and (2.8),

$$
\lim _{n \rightarrow \infty} h_{X_{n}}\left(x_{0}\right)=h_{X}\left(x_{0}\right)
$$

The theorem is proved.

REMARK 1. From the proof of this theorem one can see that $\lim _{n \rightarrow \infty} h_{X_{n}}=h_{X}$ uniformly on compacta.

2. The proof can be repeated for $h_{X}(x, \mathcal{H})$, where $\mathcal{H}$ is any bounded homogeneous domain for which $(1-\delta) \mathcal{H} \Subset \mathscr{H}$ for any $\delta>0$. For instance, if $\mathcal{H}=U^{n}$, the theorem is true.

3. Behaviour of $h_{D}$ near the boundary of a strictly pseudoconvex domain. In this section $h_{D}(z)$ means $h_{D}\left(z, B^{n}\right)$. The main purpose of this section is to prove the following.

THEOREM 3.1. Let $D$ be a bounded strictly pseudoconvex domain, $\partial D \in C^{3}$. Then $\lim _{z \rightarrow \partial D} h_{D}(z)=0$.

The next example shows that the ball $B^{n}$ may be exhausted by biholomorphic images of half of the ball. The idea of this example will be used to prove Theorem 3.1.

EXAMPLE 3.2. Let $D=B^{n} \cap\left\{z \mid \operatorname{Re} z_{1}>0\right\}$. Let $\left\{\lambda_{k}\right\}_{k=1}^{\infty} \subset D$ be any sequence of points with the properties $\lambda_{k}=\alpha_{k} e_{1}$ and $\lim _{k \rightarrow \infty} \lambda_{k}=e_{1}$, where $\alpha_{k} \in \mathbf{R},\left|\alpha_{k}\right|<1$, $e_{1}=(1,0, \ldots, 0)$.

We will prove that there exists a sequence $\left\{F_{k}\right\}, 1 \leqslant k<\infty$, of mappings $F_{k}$ : $D \rightarrow B^{n}$ with the properties:

(i) $F_{k}: D \rightarrow F_{k}(D)$ is biholomorphic for any $k<\infty$. 
(ii) $F_{k}\left(\lambda_{k}\right)=0$ for any $k<\infty$.

(iii) $\left\{F_{k}(D)\right\}_{k=1}^{\infty}$ exhausts $B^{n}$.

For this purpose let us put $z^{\prime}=\left(z_{2}, \ldots, z_{n}\right), z=\left(z_{1}, z^{\prime}\right), w=\left(w_{1}, w^{\prime}\right)$ and

$$
F_{k}:\left\{\begin{array}{l}
w_{1}=\left(z_{1}-\alpha_{k}\right) /\left(1-z_{1} \alpha_{k}\right) \\
w^{\prime}=\sqrt{1-\alpha_{k}^{2}} z^{\prime} /\left(1-z_{1} \alpha_{k}\right)
\end{array}\right.
$$

$F_{k}$ is an automorphism of the unit ball carrying $\lambda_{k}$ to $0 . F_{k}: D \rightarrow B^{n}$ and (i) and (ii) evidently hold. Now let $|w| \leqslant R<1$. From (3.1),

$$
F_{k}^{-1}:\left\{\begin{array}{l}
z_{1}=\left(w_{1}+\alpha_{k}\right) /\left(1+w_{1} \alpha_{k}\right) \\
z^{\prime}=\sqrt{1-\alpha_{k}^{2}} w^{\prime} /\left(1+w_{1} \alpha_{k}\right)
\end{array}\right.
$$

Now

$$
\begin{aligned}
|z|^{2} & =\left|z_{1}\right|^{2}+\left|z^{\prime}\right|^{2}=\frac{\left|w_{1}\right|^{2}+\alpha_{k}^{2}+w_{1} \alpha_{k}+\bar{w}_{1} \alpha_{k}+\left(1-\alpha_{k}^{2}\right)\left|w^{\prime}\right|^{2}}{\left|1+w_{1} \alpha_{k}\right|^{2}} \\
& =\frac{\left(\alpha_{k}^{2}\left|w_{1}\right|^{2}+w_{1} \alpha_{k}+\bar{w}_{1} \alpha_{k}+1\right)-\left(1-\alpha_{k}^{2}\right)\left(1-\left|w_{1}\right|^{2}-\left|w^{\prime}\right|^{2}\right)}{\left|1+w_{1} \alpha_{k}\right|^{2}} \\
& =1-\left(1-\alpha_{k}^{2}\right)\left(1-R^{2}\right) /\left|1+w_{1} \alpha_{k}\right|^{2}<1 .
\end{aligned}
$$

For $\operatorname{Re} z_{1}$, we have

$$
\begin{aligned}
1-\operatorname{Re} z_{1} & =\operatorname{Re}\left(1-\frac{w_{1}+\alpha_{k}}{1+w_{1} \alpha_{k}}\right)=\operatorname{Re} \frac{1+w_{1} \alpha_{k}-w_{1}-\alpha_{k}}{1+w_{1} \alpha_{k}} \\
& =\operatorname{Re} \frac{\left(1-\alpha_{k}\right)\left(1-w_{1}\right)}{1+w_{1} \alpha_{k}} \leqslant \frac{\left(1-\alpha_{k}\right)(1+R)}{1-R} .
\end{aligned}
$$

Thus

$$
\operatorname{Re} z_{1} \geqslant 1-\left(1-\alpha_{k}\right)(1+R) /(1-R) \text {. }
$$

From (3.2) and (3.3) one can see that, if $\alpha_{k}$ is close enough to 1 , then $z \in D$. This shows that, for a large number $N, F_{k}(D) \supset\{w|| w \mid<R\}$ if $k>N$. This proves (iii).

The idea of the proof of Theorem 3.1 is as follows. We notice that it is enough to show that for any sequence $\left\{\lambda_{k}\right\} \subset D$, if $\lambda_{k} \rightarrow z^{0} \in \partial D$, then $\lim _{k \rightarrow \infty} h_{D}\left(\lambda_{k}\right)=0$. First we reduce this statement to a similar one concerning $D_{\delta}=D \cap U_{\delta}, U_{\delta}=\{z \mid$ $\left.\left|z-z^{0}\right|<\delta\right\}$ where $\delta>0$ is any positive number. Then using the idea of Example 3.2 we find a sequence of mappings $F_{k}: D_{\delta} \rightarrow B^{n}$ with the same properties. Using then Theorem 2.1 we prove the statement $\lim _{k \rightarrow \infty} h_{D_{\delta}}\left(\lambda_{k}\right)=0$.

I. We will now use Royden's definition of the Kobayashi metric (for details, see [10]). Let $p$ be a point on a complex manifold $X$ and let $v$ be a vector on the tangent space $T_{p}(X)$ of $X$. Consider all holomorphic mappings $f: B_{R}^{1} \rightarrow X\left(B_{R}^{1}\right.$ is a disk in $C^{1}$ of radius $R$ ) satisfying the conditions $f(0)=p, f^{\prime}(0)=v$. Define the functional

$$
\Phi(p, v)=\frac{1}{\sup \left\{R: \exists f \in \mathcal{O}\left(B_{R}^{1}, X\right), f(0)=p, f^{\prime}(0)=v\right\}} .
$$


The distance $\rho\left(x_{1}, x_{2}\right)$ can be obtained by the formula

$$
\rho\left(x_{1}, x_{2}\right)=\inf \int_{0}^{1} \Phi\left(\gamma(t), \gamma^{\prime}(t)\right) d t,
$$

where the inf is taken over all piecewise smooth curves $\gamma:[0 ; 1] \rightarrow X$ such that $\gamma(0)=x_{1}$ and $\gamma(1)=x_{2}$.

The following estimate is a lemma of H. L. Royden (Lemma 2 in [10], a proof is given in [6]). We will need it in the following form.

LEMMA 3.3. Let $D \subset \mathbf{C}^{n}$ be any bounded domain, $z_{0} \in \partial D, U$ a neighbourhood of $z_{0}$, and $d=U \cap D$. There exists $\delta>0$ such that, for any point $p \in d \cap U_{\delta}, U_{\delta}=\{z \mid$ $\left.\left|z-z_{0}\right|<\delta\right\}$ and any $v \in T_{p}(D)$,

$$
\Phi_{D}(p, v) \geqslant C \Phi_{d}(p, v)
$$

where $C>0$ is a constant independent of $p$.

Lemma 3.4. Let $D \subset \mathbf{C}^{n}$ be a bounded strictly pseudoconvex domain with $\partial D \in C^{2}$, $z_{0} \in \partial D$, and $d=U \cap D$, where $U \ni z_{0}$ is a neighbourhood of $z_{0}$. If $\lim _{z \rightarrow z_{0}} h_{d}(z)=$ 0 , then $\lim _{z \rightarrow z_{0}} h_{D}(z)=0$.

Proof. (1) Let $\lambda \in d \cap U_{\delta}$, where $U_{\delta}$ is as in Lemma 3.3. Let $B_{d}(\lambda, r)$ be a ball in the Kobayashi metric such that $B_{d}(\lambda, r) \subset U_{\delta}$, and let $z \in\left[D \backslash B_{d}(\lambda, r)\right] \cap U_{\delta}$. One can see from Lemma 3.3 and Royden's definition of the Kobayashi metric that $\rho_{D}(\lambda, z) \geqslant C r$. Hence

$$
B_{D}(\lambda,(C / 2) r) \subset B_{d}(\lambda, r) .
$$

(2) Let $\left\{\lambda_{k}\right\} \subset D$ be any sequence such that $\lim _{k \rightarrow \infty} \lambda_{k}=z_{0}$. Without loss of generality we may assume that $\left\{\lambda_{k}\right\}_{k=1}^{\infty} \subset U_{\delta}$. The condition $\lim _{k \rightarrow \infty} h_{d}\left(\lambda_{k}\right)=0$ shows that there exists a sequence $\left\{R_{k}\right\}$, where $R_{k} \rightarrow \infty$ as $k \rightarrow \infty$, such that each ball $B_{d}\left(\lambda_{k}, R_{k}\right)$ can be covered by the image of an appropriate biholomorphic imbedding $F_{k}: B^{n} \rightarrow d$. From the behaviour of the Kobayashi metric near the point $z_{0}$ (for details see [6]) one can deduce that there exists a sequence $\left\{r_{k}\right\}$ such that $r_{k} \leqslant R_{k}, r_{k} \rightarrow \infty$, and $B_{d}\left(\lambda_{k}, r_{k}\right) \subset U_{\delta}$. Now, for each $k=1,2, \ldots,(3.8)$ implies that

$$
B_{D}\left(\lambda_{k},(C / 2) r_{k}\right) \subset B_{d}\left(\lambda_{k}, r_{k}\right) \subset B_{d}\left(\lambda_{k}, R_{k}\right),
$$

and therefore

$$
h_{D}\left(\lambda_{k}\right) \leqslant(2 / C)\left(1 / r_{k}\right) \text {. }
$$

Since $r_{k} \rightarrow \infty$, we have proved that $\lim _{k \rightarrow \infty} h_{D}\left(\lambda_{k}\right)=0$. Since the last statement holds for any $\left\{\lambda_{k}\right\}$ such that $\lim _{k \rightarrow \infty} \lambda_{k}=z_{0}$, we have proved Lemma 3.4.

II. $D$ now means a fixed strictly pseudoconvex domain, $D=\{z \mid \psi(z)<0\}$, where $\psi(z)$ is a strictly plurisubharmonic function of class $C^{3}$ in a neighbourhood of $\bar{D}$ with $d \psi \neq 0$ on $\partial D$. Let $z^{0} \in \partial D$, and let $U$ be a neighbourhood of $z^{0}$.

(1) First let $T$ be a linear orthogonal transformation which carries $z^{0}$ onto the origin. We will denote $T(D)$ by $D, T(U)$ by $U$, and $\psi \circ T^{-1}$ by $\psi$. 
If $U$ is small enough, we have the following Taylor formula for $\psi$ in $U$ :

$$
\begin{aligned}
\psi= & \left.2 \operatorname{Re} \sum_{\nu=1}^{n} \frac{\partial \psi}{\partial z_{\nu}}\right|_{0} z_{\nu}+\left.\operatorname{Re} \sum_{\mu, \nu=1}^{n} \frac{\partial^{2} \psi}{\partial z_{\mu} \partial z_{\nu}}\right|_{0} z_{\mu} z_{\nu} \\
& +\frac{1}{2} H_{0}(\psi, z)+O\left(|z|^{3}\right),
\end{aligned}
$$

where $\frac{1}{2} H_{0}(\psi, z)$ is the Levy form.

Let us now introduce new coordinates

$$
w_{1}=\left.\sum_{\nu=1}^{n} \frac{\partial \psi}{\partial z_{\nu}}\right|_{0} z_{\nu}+\left.\frac{1}{2} \sum_{\mu, \nu=1}^{n} \frac{\partial^{2} \psi}{\partial z_{\mu} \partial z_{\nu}}\right|_{0} z_{\mu} z_{\nu}
$$

and $w^{\prime}=\left(w_{2}, \ldots, w_{n}\right)$ where $w_{2}, \ldots, w_{n}$ are coordinates in the tangent space $T_{0}(\partial D)$ at 0 . If $U$ is small enough, this transformation is biholomorphic. In new coordinates our function (which is denoted now as $\psi_{1}(w)$ ) has the following representation:

$$
\psi_{1}(w)=2 \operatorname{Re} w_{1}+\frac{1}{2} H_{0}\left(\psi_{1}, w\right)+O\left(|w|^{3}\right),
$$

where $H_{0}(\psi, w)$ is the Levy form.

(2) Now we can find a linear transformation (we again use the letters $z_{\nu}$ for new coordinates) $z_{1}=w_{1}$ and $z^{\prime}=l\left(w^{\prime}\right)$ such that in these coordinates our function $\phi(z)=\psi_{1}\left(z_{1}, l^{-1}\left(z^{\prime}\right)\right)$ has the following representation:

$$
\phi=2 \operatorname{Re} z_{1}+2 \operatorname{Re}\left[z_{1} L(\bar{z})\right]+\left|z^{\prime}\right|^{2}+R(z),
$$

where $L$ is a linear function, $z^{\prime}=\left(z_{2}, \ldots, z_{n}\right)$, and

$$
|R(z)| \leqslant C_{1}|z|^{3}, \quad C_{1}=\text { const. }
$$

We define a domain $D_{\delta}$ as the set of solutions of the following system:

$$
\left\{\begin{array}{l}
2 \operatorname{Re} z_{1}+2 \operatorname{Re}\left[z_{1} L(\bar{z})\right]+\left|z^{\prime}\right|^{2}+R(z)<0, \\
|z|^{2}<\delta
\end{array}\right.
$$

Since $\phi$ is strictly pseudoconvex,

$$
2 \operatorname{Re}\left[z_{1} L(\bar{z})\right]+\left|z^{\prime}\right|^{2} \geqslant \sigma|z|^{2}, \quad \sigma>0 .
$$

Taking (3.12) into account one can see that, for small enough $\delta>0$,

$$
|z|^{2} \leqslant C_{2}\left(-2 \operatorname{Re} z_{1}\right) \quad \text { for any } z \in D_{\delta}
$$

where $C_{2}=$ const $>0$. We assume that such a $\delta>0$ is chosen and fixed.

(3) Let $\varepsilon>0$, and consider the transformation $\Phi_{\varepsilon}: D_{\delta} \rightarrow \mathrm{C}^{n}$ described by

$$
\Phi_{\varepsilon}:\left\{\begin{array}{l}
w_{1}=\frac{2 z_{1}+\varepsilon}{2 z_{1}-\varepsilon}, \\
w^{\prime}=\frac{2 \sqrt{\varepsilon} z^{\prime}}{2 z_{1}-\varepsilon} ;
\end{array} \quad \Phi_{\varepsilon}^{-1}:\left\{\begin{array}{l}
z_{1}=\varepsilon \frac{w_{1}+1}{2\left(w_{1}-1\right)}, \\
z^{\prime}=\sqrt{\varepsilon} \frac{w^{\prime}}{w_{1}-1} .
\end{array}\right.\right.
$$


$\psi(w)=\Phi\left(\Phi_{\varepsilon}^{-1}(w)\right)$ will have the following representation:

$$
\begin{aligned}
\psi(w)= & \varepsilon \frac{\left(w_{1}+1\right)\left(\bar{w}_{1}-1\right)+\left(\bar{w}_{1}+1\right)\left(w_{1}-1\right)}{2\left|w_{1}-1\right|^{2}} \\
& +2 \operatorname{Re}\left|\varepsilon \frac{w_{1}+1}{2\left(w_{1}-1\right)} \frac{\sqrt{\varepsilon}}{\bar{w}_{1}-1} L_{1}(\bar{w})\right|+\varepsilon \frac{\left|w^{\prime}\right|^{2}}{\left|w_{1}-1\right|^{2}}+R\left(\Phi_{\varepsilon}^{-1}(w)\right) \\
= & \varepsilon \frac{\left|w_{1}\right|^{2}-1}{\left|w_{1}-1\right|^{2}}+\varepsilon \frac{\left|w^{\prime}\right|^{2}}{\left|w_{1}-1\right|^{2}}+\varepsilon \sqrt{\varepsilon} \frac{s(w, \bar{w})}{\left|w_{1}-1\right|^{2}}+R\left(\Phi_{\varepsilon}^{-1}(w)\right)
\end{aligned}
$$

where $s(w, \bar{w})$ is a polynomial. The representation of $\Phi_{\varepsilon}\left(D_{\delta}\right)$ can now be obtained from (3.13) and (3.16):

$$
\left\{\begin{array}{l}
|w|^{2}-1+\sqrt{\varepsilon} s\left(w_{1}, \bar{w}\right)+(1 / \varepsilon) R\left(\Phi_{\varepsilon}^{-1}(w)\right)\left|w_{1}-1\right|^{2}<0 \\
4 \delta\left|w_{1}-1\right|^{2}>\varepsilon\left(\varepsilon\left|w_{1}+1\right|^{2}+4\left|w^{\prime}\right|^{2}\right)
\end{array}\right.
$$

(4) We will need below the statement: for any $\varepsilon>0$ small enough,

$$
|w|=\left|\Phi_{\varepsilon}(z)\right|<C_{3}=\text { const for any } z \in D_{\delta} .
$$

To prove this we use the representation (3.15):

$$
\begin{gathered}
\left|w_{1}\right|=\left|1+\frac{2 \varepsilon}{2 z_{1}-\varepsilon}\right| \leqslant 1+\frac{2 \varepsilon}{\left|2 \operatorname{Re} z_{1}-\varepsilon\right|} \leqslant 1+\frac{2 \varepsilon}{0+\varepsilon}=3, \\
\left|w^{\prime}\right|=\frac{2 \sqrt{\varepsilon}\left|z^{\prime}\right|}{\left|2 z_{1}-\varepsilon\right|} \leqslant \frac{2 \sqrt{\varepsilon} \sqrt{C_{2}\left(-2 \operatorname{Re} z_{1}\right)}}{\left(-2 \operatorname{Re} z_{1}\right)+\varepsilon} \leqslant \sqrt{C_{2}} .
\end{gathered}
$$

(Here we have used the inequality $2 \operatorname{Re} z_{1}<0$ and (3.14).) From (3.19) and (3.20) we get (3.18).

(5) Next we prove that

$$
\left|(1 / \varepsilon) R\left(\Phi_{\varepsilon}^{-1}(w)\right)\right| w_{1}-\left.1\right|^{2} \mid \leqslant C_{4} \sqrt{\varepsilon} .
$$

Using (3.15), (3.12) and (3.14), we obtain

$$
\begin{aligned}
\left|\frac{1}{\varepsilon} R\left(\Phi_{\varepsilon}^{-1}(w)\right)\right| w_{1}-\left.1\right|^{2} \mid & \leqslant \frac{1}{\varepsilon} C_{1}|z|^{3}\left|\frac{2 \varepsilon}{2 \operatorname{Re} z_{1}-\varepsilon}\right|^{2} \\
& \leqslant 4 C_{1} C_{2}^{3 / 2} \frac{\left(-2 \operatorname{Re} z_{1}\right)^{3 / 2} \varepsilon}{\left(-2 \operatorname{Re} z_{1}+\varepsilon\right)^{2}} \leqslant C_{4} \sqrt{\varepsilon} .
\end{aligned}
$$

The last inequality can easily be obtained by finding that the last but one expression has its maximum (for $-2 \operatorname{Re} z_{1}>0$ ) when $-2 \operatorname{Re} z_{1}=3 \varepsilon$.

(6) $\Phi_{\varepsilon}\left(D_{\delta}\right)$ now has the following description (from (3.17)):

$$
\left\{\begin{array}{l}
|w|^{2}-1+\sqrt{\varepsilon} A(w)<0 \\
4 \delta\left|w_{1}-1\right|>\varepsilon\left(\varepsilon\left|w_{1}+1\right|+4\left|w^{\prime}\right|^{2}\right)
\end{array}\right.
$$


where $|A(w)| \leqslant C_{5}=$ const. From (3.18) it follows that

$$
\Phi_{\varepsilon}\left(D_{\delta}\right) \rightarrow B^{n} \quad \text { as } \varepsilon \rightarrow 0 .
$$

The first limit in (3.23) is understood as a limit of sets.

(7) Let us return now to the initial domain $D$. Let $\lambda_{k} \rightarrow z^{0} \in \partial D$. For each $\lambda_{k}$ we choose $z^{k} \in \partial D$ to be one of the points on $\partial D$ closest to $\lambda_{k}$. Without loss of generality we may assume that for each $\lambda_{k}$ such a point $z^{k}$ is unique. We can now change the coordinate system so that the line joining $z^{k}$ and $\lambda^{k}$ is the $\operatorname{Re} z_{1}$-axis and $z^{k}$ is the origin. Then we can obtain the representation (3.11). Let the image of $\lambda_{k}$ after all these transformations be $-2 \varepsilon_{k} e_{1}$ where $\varepsilon_{k}>0$. Now we can consider the transformation $\Phi_{2 \varepsilon_{k}}$ (see (3.15)). One can see that all the constants $\delta, C_{1}-C_{5}$ are uniformly bounded for all $k \geqslant N$ for some $N$. Without loss of generality we may assume that it holds for all $k$. Now from (3.23),

$$
\Phi_{2 \varepsilon_{k}}\left(D_{\delta}\right) \rightarrow B^{n} \text { in the sense of a limit of sets. }
$$

From (3.15) with $\varepsilon$ replaced by $2 \varepsilon_{k}$,

$$
\Phi_{2 \varepsilon_{k}}\left(\lambda_{k}\right)=0 \text {. }
$$

(8) We have obtained the following. There exist a neighbourhood $U \ni z^{0}$ and a sequence $\left\{F_{k}\right\}$ of biholomorphic transformations of $d=D \cap U$ such that

(3.26) $F_{k}\left(\lambda_{k}\right)=0$,

(3.27) $F_{k}(d) \rightarrow B^{n}$ uniformly in the sense of the limit of sets.

(9) It is clear from (3.27) that for each $k$ there exists a number $\delta_{k}>0, \lim _{k \rightarrow \infty} \delta_{k}$ $=0$, such that if we set $T_{k}=\left(1-\delta_{k}\right) F_{k}$ then

(i) $T_{k}(d) \subset B^{n}$ for each $k$,

(ii) $\left\{T_{k}(d)\right\}$ exhausts $B^{n}$.

Now (3.26) implies that $T_{k}\left(\lambda_{k}\right)=0$, and Theorem 2.1 yields

$$
\lim _{k \rightarrow \infty} h_{d}\left(\lambda_{k}\right)=\lim _{k \rightarrow \infty} h_{T_{k}(d)}(0)=h_{B^{n}}(0)=0 .
$$

(10) Theorem 3.1 now follows from (3.28) and Lemma 3.4. This completes the proof of Theorem 3.1 .

\section{Proofs of Theorems I and II, and remarks.}

1. Proof of Theorem I. Let $F_{n}: D \rightarrow X, n=1,2, \ldots$, be a sequence of biholomorphic imbeddings such that, for any compact $K \subset X$, there exists $N$ such that $F_{n}(D) \supset K$ for all $n>N$. The existence of such a sequence follows from the conditions of the theorem. Let $x_{0} \in X$. Without loss of generality we may assume that $F_{n}(D) \ni x_{0}$ for every $n$. We now consider the set $A=\left\{F_{n}^{-1}\left(x_{0}\right)\right\} \subset D$. There are two cases to be considered.

Case $1 . A$ is relatively compact in $D$. Then by Lemma $1.1 X$ is biholomorphically equivalent to $D$.

Case 2. $A$ has at least one limit point $z^{0} \in \partial D$. Without loss of generality (by passing to a subsequence if necessary) we may suppose that $\lim _{n \rightarrow \infty} F_{n}^{-1}\left(x_{0}\right)=z^{0}$. From Theorem 3.1 it follows that $\lim _{n \rightarrow \infty} h_{D}\left(F_{n}^{-1}\left(x_{0}\right)\right)=0$. Now Theorem 2.1 and the fact that $h$ is invariant imply that

$$
h_{X}\left(x_{0}\right)=\lim _{n \rightarrow \infty} h_{F_{n}(D)}\left(x_{0}\right)=\lim _{n \rightarrow \infty} h_{D}\left(F_{n}^{-1}\left(x_{0}\right)\right)=0 .
$$


Theorem 1.3 (for $\mathcal{H}=B^{n}$ ) now shows that $X$ is biholomorphically equivalent to $B^{n}$.

2. Proof of Theorem II. Let $D_{1}$ and $D_{2}$ be the two pseudoconvex domains. Since $D_{2}$ can be exhausted by biholomorphic images of $D_{1}$ it follows from Theorem I that either $D_{2}$ is biholomorphically equivalent to $D_{1}$, or $D_{2}$ is biholomorphically equivalent to $B^{n}$. In the latter case, since $D_{1}$ can be exhausted by $D_{2}$, it follows from Theorem I that $D_{1}$ is biholomorphically equivalent to $B^{n}$ and, therefore, to $D_{2}$. Theorem II is proved.

Let $D \subset C^{n}$ be a bounded domain. Let $z^{0} \in \partial D$ have a neighbourhood $U$ such that $\partial D \cap U$ is strictly pseudoconvex and of class $C^{3}$. It follows as in the proof of Theorem 3.1 that

$$
\lim _{z \rightarrow z^{0}} h_{D}(z)=0 \text {. }
$$

(In fact this very statement was proved in section 3; it follows from Lemma 3.4 and (3.28) for an appropriate $d \subset D \cap U$.)

We now denote by $\operatorname{Aut}(D)$ the group of analytical automorphisms of $D$. If $z^{\prime} \in D, \Gamma\left(z^{\prime}\right)=\left\{z \mid z=F\left(z^{\prime}\right), F \in \operatorname{Aut}(D)\right\}$ is the orbit of $z^{\prime}$. If we assume that the closure $\overline{\Gamma\left(z^{\prime}\right)}$ contains $z^{0}$, then there exists a sequence $\left\{F_{k}\right\} \subset \operatorname{Aut}(D)$ such that $\lim _{k \rightarrow \infty} F_{k}\left(z^{\prime}\right)=z^{0}$. Now using (4.1) we obtain

$$
h_{D}\left(z^{\prime}\right)=\lim _{k \rightarrow \infty} h_{D}\left(F_{k}\left(z^{\prime}\right)\right)=0 .
$$

Hence it follows from Theorem 1.3 that $D$ is biholomorphically equivalent to $B^{n}$. We have proved the following statement.

Lemma 4.1. Let $D \subset \mathbf{C}^{n}$ be a bounded domain. Let $z^{0} \in \partial D$ have a neighbourhood $U$ such that $\partial D \cap U$ is strictly pseudoconvex and belongs to the class $C^{3}$. If there exists a point $z^{\prime} \in D$ such that the closure of its orbit $\Gamma\left(z^{1}\right)$ contains $z^{0}$, then $D$ is biholomorphically equivalent to $B^{n}$.

As a corollary of Lemma 4.1 we obtain a new proof of the following well-known theorem (for $\partial D \in C^{\infty}$, see [11]):

THEOREM 4.2. Let $D$ be a bounded strictly pseudoconvex domain with $\partial D \in C^{3}$. If $\operatorname{Aut}(D)$ is noncompact, then $D$ is biholomorphically equivalent to $B^{n}$.

More information about $\operatorname{Aut}(D)$ can be found in [7].

If $G$ is a bounded domain, $z^{0} \in \partial G$, and $z^{0}$ has a neighbourhood on $\partial G$ which is similar to a part of the boundary of the unit polydisk $U^{n}$, we can prove an analogue of Theorem 3.1. More precisely, let there be an analytic function $f(z)$ in a neighbourhood $U$ of $z^{0}$ such that

$$
\partial G \cap U=\{z|| f(z) \mid=1\} .
$$

Using the functions $h_{G}\left(x, U^{n}\right)$ in place of the $h_{G}\left(x, B^{n}\right)$ we find that $\lim _{z \rightarrow z^{0}} h_{G}\left(z, U^{n}\right)=0$. Analogously to Lemma 4.1 one can obtain

LEMMA 4.3. Let $D \subset \mathrm{C}^{n}$ be a bounded domain. Let $z^{0} \in \partial G$ have a neighbourhood $U$ with property (4.2) ( for some analytic function $f$ ). If for some point $z^{\prime} \in G$ the closure of the orbit (with respect to Aut $G$ ) of $z^{\prime}$ contains $z^{0}$, then $G$ is biholomorphically equivalent to $U^{n}$. 
Now let $G \subset \mathbf{C}^{n}$ be a bounded polyhedron. Then $G$ is a bounded domain such that $G=\left\{z \in \mathbf{C}^{n}|| f_{s} \mid<1, s=1, \ldots, p\right\}$, where the functions $f_{1}, \ldots, f_{p}$ are holomorphic in a neighbourhood of the closure of $G$. Then the $(2 n-1)$-dimensional measure of all points having no neighbourhood $U$ with property (4.2) is zero. Let

$$
M(G)=\bigcup_{z \in G} \overline{\Gamma(z)} \cap \partial G
$$

and let $\mu_{2 n-1}(M)$ be the $(2 n-1)$-dimensional measure of $M$. If $G$ is the polydisk $U^{n}$, then evidently $M(G)=\partial U^{n}$.

Lemma 4.3 yields

THEOREM 4.4. If $G$ is a bounded polyhedron and $\mu_{2 n-1}(M(G))>0$ then $G$ is biholomorphically equivalent to $U^{n}$.

Biholomorphic classification of polyhedra and Aut $G$ were considered in [5].

\section{BIBLIOGRAPHY}

1. H. Alexander, Extremal holomorphic imbeddings between ball and polydisc, Proc. Amer. Math. Soc. 68 (1978), 200-202.

2. J.-E. Fornaess and E. L. Stout, Polydiscs in complex manifolds, Math. Ann. 227 (1977), 145-153.

3. J.-E. Fornaess and Sibony Nessim, Increasing sequences of complex manifolds, Math. Ann. 255 (1981), 351-360.

4. B. L. Fridman, On the imbedding of a strictly pseudoconvex domain in a polyhedron, Dokl. Akad. Nauk SSSR 249 (1979) = Soviet Math. Dokl. 20 (1979), 1228-1232.

5. __ On a class of analytic polyhedra, Dokl. Akad. Nauk SSSR 242 (1978) = Soviet Math. Dokl. 19 (1979), 1258-1261.

6. Ian Graham, Boundary behaviour of the Caratheodory and Kobayashi metrics on strongly pseudoconvex domains in $\mathbf{C}^{n}$ with smooth boundary, Trans. Amer. Math. Soc. 207 (1975), 219-240.

7. Robert E. Greene and Steven G. Krantz, The automorphism groups of strongly pseudoconvex domains (to appear).

8. P. Klembeck, Kähler metrics of negative curvature, the Bergman metric near the boundary and the Kobayashi metric on smooth bounded strictly pseudoconvex sets, Indiana Univ. Math. J. 27 (1978), 275-282.

9. S. Kobayashi, Hyperbolic manifolds and holomorphic mappings, Dekker, New York, 1970.

10. H. L. Royden, Remarks on the Kobayashi metric, Lecture Notes in Math., vol. 185, Springer-Verlag, Berlin and New York, 1971, pp. 125-137.

11. B. Wong, Characterization of the unit ball in $\mathbf{C}^{n}$ by its automorphism group, Invent. Math. 41 (1977), 253-257.

Department of Mathematics, University of Michigan, Flint, Michigan 48503

Current address: Department of Mathematics, Wichita State University, Wichita, Kansas 67208 